\title{
No association between polymorphisms of WNT2 and schizophrenia in a Korean population
}

\author{
Hak-Jae Kim', Jin Kyung Park², Su Kang Kim³, Sung Wook Kang³, Jong Woo Kim², Hyun-Kyung Park4, Ah-Rang Cho², \\ Ji Young Song 2 and Joo-Ho Chung*3
}

\begin{abstract}
Background: Wingless-type MMTV integration site family member 2 (WNT2) has a potentially important role in neuronal development; however, there has yet to be an investigation into the association between single nucleotide polymorphisms (SNPS) of WNT2 and schizophrenia. This study aimed to determine whether certain SNPs of WNT2 were associated with schizophrenia in a Korean population.
\end{abstract}

Methods: e genotyped 7 selected SNPs in the WNT2 gene region (approximately $46 \mathrm{~Kb}$ ) using direct sequencing in 288 patients with schizophrenia and 305 healthy controls.

Results: Of the SNPs examined, one SNP showed a weak association with schizophrenia ( $p=0.017$ in the recessive model). However, this association did not remain statistically significant after Bonferroni correction.

Conclusion: The present study does not support a major role for WNT2 in schizophrenia. This could be due to the size of the population. Therefore, additional studies would be needed to definitively rule out the gene's minor effects.

\section{Background}

Schizophrenia is a complex disease that develops in approximately $0.5-1.0 \%$ of the global population and inflicts physical suffering and mental anguish on the affected individuals and their families $[1,2]$. The neurodevelopmental theory of schizophrenia postulates that abnormal neurodevelopment is one of the etiological factors of schizophrenia [3-6]. Recent studies suggest that schizophrenia may be a subtle disorder of brain development or plasticity [7-9].

Wnt proteins belong to a family of molecules that locally activate cell signaling pathways, regulating cell fate and playing an important role in development [10]. Evidence suggests that Wnt signaling and insulin signaling, which regulates glycogen synthase kinase-3 (GSK-3), may be important in schizophrenia [11]. Several lines of evidence support the association between signaling aberrations and schizophrenia. However, research has shown changes in gene expressions in schizophrenia [12-16], and, in particular, that some such changes affect GSK-3 [14,17-19]. Researchers have also found Wnt signaling

* Correspondence: jhchung@khu.ac.kr

3 Kohwang Medical Research Institute, School of Medicine, Kyung Hee University, Seoul 130-701, Republic of Korea

Full list of author information is available at the end of the article abnormalities associated with schizophrenia. For example, expressions of $\beta$-catenin proteins decreased in the brains of schizophrenia patients, presumably due to aberrant regulation of $\beta$-catenin degradation mediated by activated GSK-3 $\beta[20,21]$. Another report suggested the enhanced GSK-3 $\beta$ activity was secondary to diminished phosphorylation by Akt, since there is decreased Akt expression in schizophrenia patients' brains [22]. Furthermore, researchers found increased Wnt-1 expression in the hippocampi of schizophrenia patients relative to normal subjects. Wnt-1 is an important molecule in the Wnt pathway [23]. Several studies have implicated Wnt signaling genes in bipolar disorder, as well as in schizophrenia. Researcher found that the frizzled-3 gene (FZD3), which encodes a receptor for Wnt ligands, was associated with schizophrenia in 3 different samples [24-26].

The wingless-type MMTV integration site family, member 2 (WNT2) gene is located at chromosome $7 q 31.2$, a region that, in a genome scan study, showed evidence of a link to schizophrenia [27]. WNT2 is one of the WNT genes that are expressed in a variety of tissues during development [28]. Knockout and expression studies in mice, zebra fish, and Xenopus have demonstrated WNT genes' crucial roles in the development and pat- 
terning of the central nervous system [29-31]. WNT genes encode several signaling proteins that are essential in embryo patterning, cell proliferation, and cell determination $[32,33]$. The WNT signaling pathway also includes several receptors expressed in both developing and mature nervous systems [34].

Previously, several studies have addressed whether the WNT2 gene is associated with autism, a neurodevelopmental disorder [35-37]. Although Wassink et al. reported that one of WNT2 SNPs (rs2024233 on exon 5) is associated with autism [35], following association studies did not find any significant associations with autism $[36,37]$. In addition, Proitsi et al. performed a combined positional and candidate association screen, by identifying known WNT signaling genes, and showed that Dickkopf-4 was associated with schizophrenia [38]. There have been no published investigations of the association between WNT2 genetic polymorphisms and schizophrenia, despite its potentially important developmental roles and its importance in genetics. In this study, therefore, we investigated the association between WNT2 polymorphisms and schizophrenia in a Korean population.

\section{Methods}

\section{Subjects}

For this study, we evaluated 288 schizophrenic patients (193 males, aged $42.98 \pm 10.63$ years [mean \pm SD]; 95 females, aged $43.03 \pm 10.65)$ and 305 controls with no clinical evidence of any disorders (147 males, $39.92 \pm$ 5.82; 158 females, $36.55 \pm 6.77$ ). The schizophrenic patients met the Diagnostic and Statistical Manual of Mental Disorders, Fourth Edition (DSM-IV) [39] criteria for schizophrenia. We reviewed each patient's medical record and rated the schizophrenic patients using the Brief Psychiatric Rating Scale (BPRS) [40], the Scale for the Assessment of Negative Symptoms (SANS) [41], and the operational criteria (OPCRIT) checklist [42].

To evaluate patients for schizophrenia with poor concentration, we used the OPCRIT Checklist. Of the many OPCRIT items, we selected only a few items, already described in our previous study, for use [43]. We determined which patients to place in the poor concentration subgroup according to patients' subjective complaints of being unable to think clearly or make decisions (scoring: 1 for a duration of one week, 2 for two weeks, and 3 for one month) [42]. Thus, we examined 167 schizophrenic patients having poor concentration (116 males, $42.8 \pm$ 10.0; 51 females, $41.9 \pm 10.8)$ and 121 schizophrenic patients who had no such complaints (77 males, $43.6 \pm$ 11.2; 44 females, $44.8 \pm 10.7)$. We also analyzed the associations between the schizophrenic patients' SNPs and their SANS scores (Table 1). The genotype frequencies of rs6948009 was evaluated (A/A, n = 211; A/G, n = 71; G/ $\mathrm{G}, \mathrm{n}=3)$ for the analysis with SANS scores.
We recruited control subjects who had been found mentally fit by a general health checkup program. All studies were carried out according to the guidelines of the Declaration of Helsinki [44]. We obtained written informed consent from each subject. The study was approved by the ethics review committee of the Medical Research Institute, Kyung Hee University Medical Center, Seoul, Republic of Korea.

\section{SNP Genotyping}

We selected 8 SNPs from WNT2 genes in chromosome $7 \mathrm{q} 31.2$ by downloading all the SNPs typed in the WNT2 genes from the HapMap database (http://www.hapmap.org/;genome build 35) and dbSNP database version 129. From these, we selected tag SNPs using the Tagger program's aggressive tagging option (Paul de Bakker, http://www.broad.mit.edu/mpg/tagger/), so as to capture SNPs with a minor allele frequency of $<5 \%\left(r^{2}>0.8\right)$. This gave 2 exonic SNPs (rs2024233 and rs1051751), 3 intronic SNPs (rs733154, rs3779548, and rs17132543), and 3 regulatory SNPs (thought to be the promoter; the intervals are 2000 bp up- and downstream between the genes) (rs6948009 [869 bp downstream from the last nucleotide of the 3'-UTR], rs4730775 [164 bp downstream from the last nucleotide of the 3'-UTR], and rs39315 [219 bp upstream from the first nucleotide of the 5 '-UTR]). In addition, we chose these SNPs because they were previously evaluated for relation to schizophrenia risk or showed evidence of functional significance. Figure 1 shows the locations of the selected SNPs.

Using a commercially available Qiagen DNA Extraction kit (Qiagen, Tokyo, Japan), we extracted genomic DNA from blood samples that had been placed in EDTA. We amplified the genomic DNA using the primers shown in Table 2 for each SNP, sequenced the PCR products using an ABI Prism 377 automatic sequencer (PE Applied Biosystems, Foster City, California, USA), and analyzed the sequence data using SeqManII software (DNASTAR Inc., Madison, Wisconsin, USA).

\section{Statistical analysis}

Using SNPstats, we assessed the Hardy-Weinberg equilibrium (HWE) for both controls and schizophrenia patients [45]. For the linkage disequilibrium (LD) block, we used the Haploview version 3.32 [46]. We inferred the haplotypes and their frequencies using the EM algorithm [47]. We used the multiple logistic regression model to calculate odds ratios (OR), 95\% confidence intervals, and corresponding $p$ values (controlling age and gender as covariables), to analyze the association between schizophrenia and both SNPs and haplotypes. Furthermore, we analyzed the association between the schizophrenia subgroup and both SNPs and haplotypes, using SNPstats, the HapAnalyzer version 1.0 [48], and Helixtree (Golden 
Table 1: WNT2 SNPs genotype and allele frequencies in schizophrenia patients and healthy controls

\begin{tabular}{|c|c|c|c|c|c|c|c|c|}
\hline \multirow[b]{2}{*}{ SNP } & \multirow[b]{2}{*}{ Genotype } & \multicolumn{2}{|c|}{ Schizophrenia } & \multicolumn{2}{|c|}{ Control } & \multirow[b]{2}{*}{ Model } & \multirow[b]{2}{*}{ OR (95\% CI) } & \multirow[b]{2}{*}{ P value } \\
\hline & & Freq & $\%$ & Freq & $\%$ & & & \\
\hline rs39315 & $\mathrm{A} / \mathrm{A}$ & 90 & 0.31 & 79 & 0.26 & co-dominant & $1.21(0.95-1.54)$ & 0.12 \\
\hline \multirow[t]{2}{*}{ 5'-near gene } & $\mathrm{A} / \mathrm{G}$ & 137 & 0.48 & 150 & 0.49 & dominant & $1.38(0.94-2.02)$ & 0.1 \\
\hline & $\mathrm{G} / \mathrm{G}$ & 61 & 0.21 & 76 & 0.25 & recessive & $1.20(0.80-1.81)$ & 0.38 \\
\hline rs17132543 & $\mathrm{A} / \mathrm{A}$ & 97 & 0.34 & 98 & 0.32 & co-dominant & $1.14(0.89-1.45)$ & 0.3 \\
\hline \multirow[t]{2}{*}{ Intron4 } & $A / G$ & 138 & 0.48 & 145 & 0.48 & dominant & $1.16(0.80-1.68)$ & 0.43 \\
\hline & $\mathrm{G} / \mathrm{G}$ & 53 & 0.18 & 62 & 0.2 & recessive & $1.22(0.79-1.89)$ & 0.37 \\
\hline rs3779548 & $\mathrm{A} / \mathrm{A}$ & 127 & 0.44 & 135 & 0.44 & co-dominant & $0.97(0.74-1.25)$ & 0.8 \\
\hline \multirow[t]{2}{*}{ Intron4 } & $A / G$ & 128 & 0.44 & 136 & 0.45 & dominant & $0.99(0.70-1.40)$ & 0.96 \\
\hline & $\mathrm{G} / \mathrm{G}$ & 33 & 0.11 & 34 & 0.11 & recessive & $0.88(0.50-1.53)$ & 0.65 \\
\hline rs733154 & $\mathrm{A} / \mathrm{A}$ & 171 & 0.59 & 177 & 0.58 & co-dominant & $1.00(0.74-1.33)$ & 0.97 \\
\hline \multirow[t]{2}{*}{ Intron4 } & $A / G$ & 103 & 0.36 & 109 & 0.36 & dominant & $1.02(0.72-1.44)$ & 0.93 \\
\hline & $\mathrm{G} / \mathrm{G}$ & 14 & 0.05 & 19 & 0.06 & recessive & $0.89(0.42-1.92)$ & 0.78 \\
\hline rs2024233 & $\mathrm{A} / \mathrm{A}$ & 87 & 0.3 & 84 & 0.28 & co-dominant & $1.11(0.87-1.43)$ & 0.41 \\
\hline \multirow[t]{2}{*}{ 3'-UTR } & $A / G$ & 148 & 0.51 & 157 & 0.51 & dominant & $1.15(0.79-1.69)$ & 0.46 \\
\hline & $\mathrm{G} / \mathrm{G}$ & 53 & 0.18 & 64 & 0.21 & recessive & $1.14(0.74-1.77)$ & 0.54 \\
\hline rs4730775 & $\mathrm{G} / \mathrm{G}$ & 176 & 0.61 & 184 & 0.6 & co-dominant & $0.89(0.66-1.20)$ & 0.45 \\
\hline \multirow[t]{2}{*}{ 3'-near gene } & $\mathrm{G} / \mathrm{A}$ & 94 & 0.33 & 112 & 0.37 & dominant & $1.02(0.71-1.45)$ & 0.93 \\
\hline & $\mathrm{A} / \mathrm{A}$ & 17 & 0.06 & 9 & 0.03 & recessive & $0.35(0.14-0.85)$ & 0.017 \\
\hline
\end{tabular}

Freq, frequency; $\mathrm{OR}$, odds ratio; $\mathrm{Cl}$, confidence interval.

Helix Inc., MT, USA). We assessed the associations between SNPs and SANS scores, using one-way ANOVA to analyze the relationship between SANS scores and genotype frequencies in the schizophrenia subgroup. We calculated the power, given the sample size, using a genetic power calculator http://pngu.mgh.harvard.edu/ $\sim$ purcell/gpc [49], and, to reduce error, we adjusted the effective sample size (calculated sample size $\times 100 / 95)$.
When we calculated the sample powers, we found our case-control study was sufficiently powerful to determine a positive association. In this study, we found sample powers of 0.8046 (rs39315, effective sample size, number of cases for $80 \%$ power $=284), 0.8493(\mathrm{rs} 17132543, \mathrm{n}=$ 252), 0.9338 (rs3779548, $\mathrm{n}=188), 0.9677$ (rs733154, $\mathrm{n}=$ 155), 0.9145 (rs2024233, $\mathrm{n}=208$ ), and 0.9689 (rs4730775, $\mathrm{n}=154$ ) for detecting a two-fold increased risk, assuming

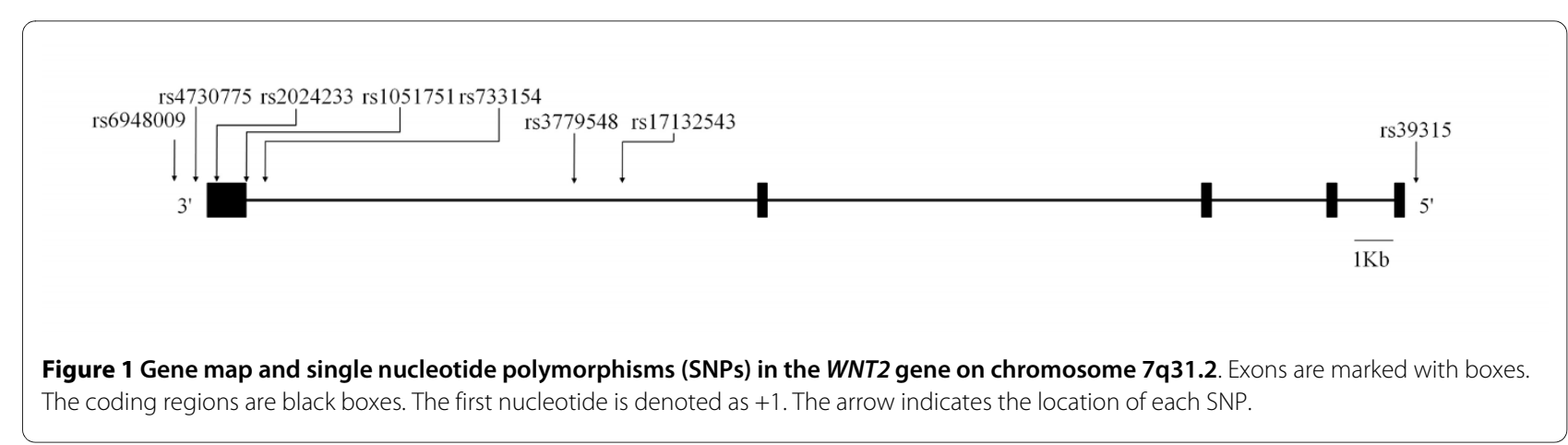


Table 2: Primer Sequences

\begin{tabular}{|c|c|c|c|}
\hline SNP & Sequence(5'-3') & Product Size & Temperature \\
\hline \multirow[t]{2}{*}{ rs39315 } & Forward CCTCCCTATGGGCTCTGTATT & 450 & 60 \\
\hline & Reverse CACGGGTGCATGAAATGATGG & & \\
\hline \multirow[t]{2}{*}{ rs17132543 } & Forward AGCCTCTAGAGAAGTCCTGAAG & 373 & 60 \\
\hline & Reverse CTCCCAACCACACTCACACACA & & \\
\hline \multirow[t]{2}{*}{ rs3779548 } & Forward GTGTGGCCTACTITGCAGAAG & 355 & 60 \\
\hline & Reverse TTCTCCAGCACCTAGACTGTG & & \\
\hline \multirow[t]{2}{*}{ rs733154 } & Forward GGATCCTTGATCGAGCAGAGCCA & 301 & 60 \\
\hline & Reverse GACTGCAGCAGGAGAGACAGTTA & & \\
\hline \multirow[t]{2}{*}{ rs1051751 } & Forward TGGGCCCACAGAACGAGTATAAC & 327 & 65 \\
\hline & Reverse CCAGAGCTTCCAGGCAGTCCT & & \\
\hline \multirow[t]{2}{*}{ rs2024233 } & Forward GTAACAAGGTGGGGACGTGTGT & 319 & 62 \\
\hline & Reverse GAGATTCCATGGGTCACATGCA & & \\
\hline \multirow[t]{2}{*}{ rs4730775 } & Forward TGGGGATACAAGATTGGTGAAC & 360 & 65 \\
\hline & Reverse GATGGCAGAAGCCAACCACTA & & \\
\hline \multirow[t]{2}{*}{ rs6948009 } & Forward GGTCATTTAGACTGAGACTCG & 461 & 60 \\
\hline & Reverse CACCAATCCCTTCGCCTCTCT & & \\
\hline
\end{tabular}

an $\alpha$-level of 0.05 . However, the sample powers in the divided schizophrenia subgroup (with or without poor concentration) were not sufficient (data not shown). We applied the Bonferroni correction by multiplying $P$ values by the number of SNPs analyzed $(n=6)$.

\section{Results}

Of the eight SNPs we examined, all were polymorphic. The genotype distributions of six SNPs (rs39315, rs17132543, rs3779548, rs733154, rs2024233, and rs4730775) were in HWE $(P>0.05)$. We did not estimate one SNP (rs6948009) for the association analysis because its genotype distribution was not in HWE $(p<0.05)$. Also, we excluded one SNP (rs1052751) from the analysis, since it was not polymorphic. Of these SNPs, one SNP (rs4730775) was weakly associated with schizophrenia. Despite this association ( $\mathrm{p}=0.017$ in the recessive model), we could not prove the statistical significance of analysis after the Bonferroni correction. Thus, these results do not support a significant role for the WNT2 sequence variation in the etiology of schizophrenia.

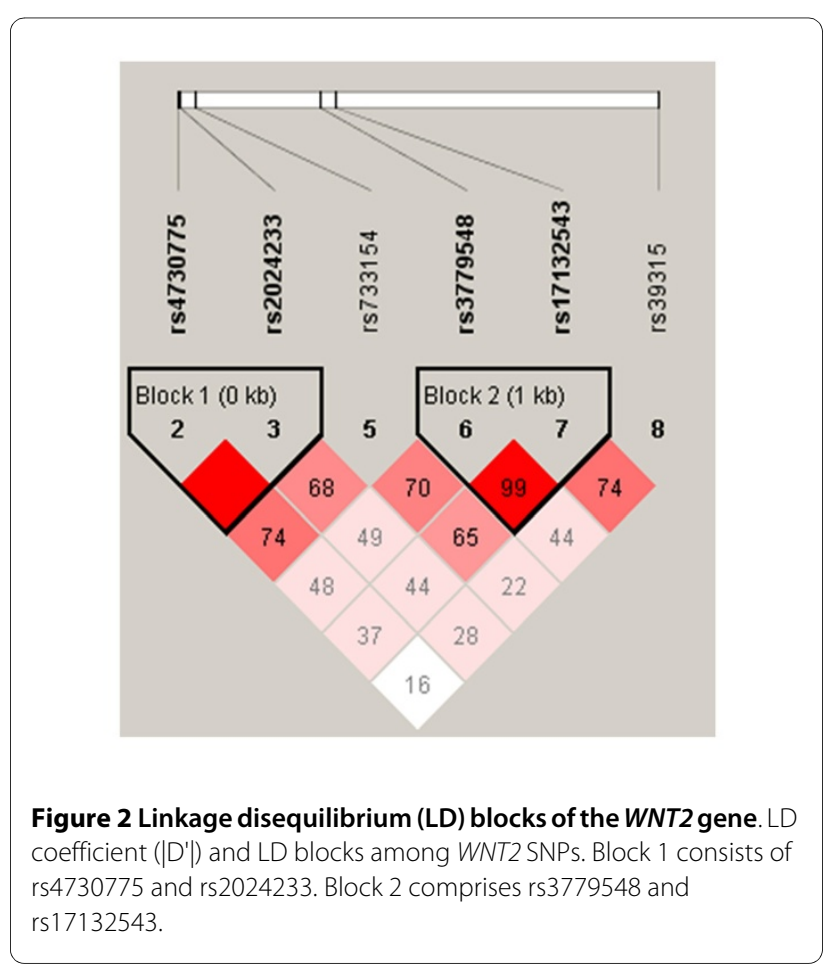


Table 3: WNT2 SNPs haplotype frequencies in schizophrenia patients and healthy controls.

\begin{tabular}{|c|c|c|c|c|c|c|c|c|c|c|}
\hline \multirow{2}{*}{$\begin{array}{c}\text { Haplotype } \\
\text { (Block 1) }\end{array}$} & \multirow[b]{2}{*}{ Type } & \multicolumn{2}{|c|}{ Schizophrenia } & \multirow[b]{2}{*}{ Freq } & \multicolumn{2}{|c|}{ Control } & \multirow[b]{2}{*}{ Freq } & \multirow[b]{2}{*}{ Models } & \multirow[b]{2}{*}{ OR(95\% Cl) } & \multirow[b]{2}{*}{$P$ value } \\
\hline & & Freq & $\%$ & & Freq & $\%$ & & & & \\
\hline & $\mathrm{HH}$ & 51 & 0.19 & & 66 & 0.21 & & co-dominant & $0.92(0.73-1.16)$ & 0.4778 \\
\hline \multirow[t]{3}{*}{ HAP1 GG } & $\mathrm{H}-$ & 142 & 0.52 & 0.44 & 163 & 0.51 & 0.46 & dominant & $0.91(0.64-1.31)$ & 0.6236 \\
\hline & -- & 82 & 0.3 & & 89 & 0.28 & & recessive & $0.87(0.58-1.31)$ & 0.5004 \\
\hline & $\mathrm{HH}$ & 31 & 0.11 & & 37 & 0.12 & & co-dominant & $1.04(0.82-1.32)$ & 0.7506 \\
\hline \multirow[t]{3}{*}{ HAP2 GA } & $\mathrm{H}-$ & 121 & 0.44 & 0.33 & 132 & 0.42 & 0.32 & dominant & $1.09(0.79-1.51)$ & 0.604 \\
\hline & -- & 123 & 0.45 & & 149 & 0.47 & & recessive & $0.96(0.58-1.60)$ & 0.8901 \\
\hline & $\mathrm{HH}$ & 15 & 0.05 & & 11 & 0.03 & & co-dominant & $1.07(0.81-1.42)$ & 0.6325 \\
\hline \multirow[t]{2}{*}{ HAP3 AA } & $\mathrm{H}-$ & 93 & 0.34 & 0.22 & 113 & 0.36 & 0.21 & dominant & $1.01(0.73-1.41)$ & 0.9446 \\
\hline & -- & 167 & 0.61 & & 194 & 0.61 & & recessive & $1.61(0.73-3.57)$ & 0.2405 \\
\hline \multirow{3}{*}{$\begin{array}{l}\text { Haplotype } \\
\text { (Block 2) }\end{array}$} & & Schiz & renia & & & & & & & \\
\hline & Type & Freq & $\%$ & Freq & Freq & $\%$ & Freq & Models & OR(95\% Cl) & $P$ value \\
\hline & $\mathrm{HH}$ & 50 & 0.18 & & 65 & 0.2 & & co-dominant & $0.90(0.72-1.14)$ & 0.3894 \\
\hline \multirow[t]{3}{*}{ HAP1 AG } & $\mathrm{H}-$ & 130 & 0.47 & 0.42 & 152 & 0.48 & 0.44 & dominant & $0.88(0.63-1.24)$ & 0.4723 \\
\hline & -- & 95 & 0.35 & & 101 & 0.32 & & recessive & $0.86(0.57-1.30)$ & 0.4881 \\
\hline & $\mathrm{HH}$ & 31 & 0.11 & & 35 & 0.11 & & co-dominant & $1.01(0.80-1.29)$ & 0.912 \\
\hline \multirow[t]{3}{*}{ HAP2 GA } & $\mathrm{H}-$ & 123 & 0.45 & 0.34 & 142 & 0.45 & 0.33 & dominant & $1.01(0.73-1.40)$ & 0.9338 \\
\hline & -- & 121 & 0.44 & & 141 & 0.44 & & recessive & $1.03(0.62-1.72)$ & 0.9181 \\
\hline & $\mathrm{HH}$ & 15 & 0.05 & & 14 & 0.04 & & co-dominant & $1.12(0.86-1.48)$ & 0.4011 \\
\hline \multirow[t]{2}{*}{ HAP3 AA } & $\mathrm{H}-$ & 104 & 0.38 & 0.24 & 114 & 0.36 & 0.22 & dominant & $1.13(0.82-1.57)$ & 0.4568 \\
\hline & -- & 156 & 0.57 & & 190 & 0.6 & & recessive & $1.25(0.59-2.64)$ & 0.5543 \\
\hline
\end{tabular}

Freq, frequency; OR, odds ratio; $\mathrm{Cl}$, confidence intervals.

To further analyze the haplotype structure in our samples, we characterized the two LD blocks between the six WNT2 SNPs (including the 5'UTR and 3'UTR) in the control subjects, using the pairwise D'values (Figure 2). Per the criteria used by Gabriel et al. [50], the LD data revealed two haplotype blocks across $W N T 2$. The D' values from rs4730775 to rs32024233 and from rs37795488 to rs17132543 ranged between 0.95 and 0.99 , indicating strong LD between each pair of markers (Figure 2). We performed a haplotype-based association analysis between the schizophrenic and control groups for different combinations of SNPs within WNT2, revealing two LD blocks (block 1, rs4730775 and rs32024233; block 2, rs37795488 and rs17132543). However, we did not detect significant associations between the haplotypes in block 1 or 2 and schizophrenia (all p-values of the analysis $>0.05$; Table 3). We then assessed the gene and haplotype associations with these SNPs in the poor-concentration schizophrenia subgroup (determined using the OPCRIT Checklist). In this subgroup, the genotype distributions of the seven SNPs considered were in HWE $(p>0.05)$. We then analyzed the genetic associations of these SNPs within the subgroup. The SNPs of the WNT2 gene were not associated with poor concentration, a clinical symptom of schizophrenia (Table 4). In addition, we assessed the associations between SNPs and SANS scores. No statistically significant differences appeared in the total SANS scores among the seven SNPs (Table 5). To compare our genotypic results with different ethnic populations, we searched the human SNP database (http:// 
Table 4: WNT2 genotype frequencies in Korean schizophrenia patients with poor concentration

\begin{tabular}{|c|c|c|c|c|c|c|c|c|}
\hline \multirow[b]{2}{*}{ SNP } & \multirow[b]{2}{*}{ Genotype } & \multicolumn{2}{|c|}{ NPC } & \multicolumn{2}{|c|}{ PC } & \multirow[b]{2}{*}{ Model } & \multirow[b]{2}{*}{ OR (95\% Cl) } & \multirow[b]{2}{*}{$P$ value } \\
\hline & & Freq & $\%$ & Freq & $\%$ & & & \\
\hline rs39315 & $\mathrm{A} / \mathrm{A}$ & 40 & 33.1 & 50 & 29.9 & co-dominant & $0.97(0.70-1.34)$ & 0.85 \\
\hline \multirow[t]{2}{*}{ 5'-near gene } & $A / G$ & 52 & 43.0 & 85 & 50.9 & dominant & $1.16(0.70-1.92)$ & 0.57 \\
\hline & $\mathrm{G} / \mathrm{G}$ & 29 & 24.0 & 32 & 19.2 & recessive & $0.75(0.43-1.33)$ & 0.33 \\
\hline rs17132543 & $A / A$ & 40 & 33.1 & 57 & 34.1 & co-dominant & $0.99(0.71-1.38)$ & 0.95 \\
\hline \multirow[t]{2}{*}{ Intron4 } & $A / G$ & 59 & 48.8 & 79 & 47.3 & dominant & $0.96(0.59-1.58)$ & 0.88 \\
\hline & $\mathrm{G} / \mathrm{G}$ & 22 & 18.2 & 31 & 18.6 & recessive & $1.02(0.56-1.87)$ & 0.95 \\
\hline rs3779548 & $A / A$ & 50 & 41.3 & 77 & 46.1 & co-dominant & $0.90(0.63-1.27)$ & 0.54 \\
\hline \multirow[t]{2}{*}{ Intron4 } & $A / G$ & 57 & 47.1 & 71 & 42.5 & dominant & $0.82(0.51-1.32)$ & 0.42 \\
\hline & $\mathrm{G} / \mathrm{G}$ & 14 & 11.6 & 19 & 11.4 & recessive & $0.99(0.47-2.06)$ & 0.97 \\
\hline rs733154 & $A / A$ & 67 & 55.4 & 104 & 62.3 & co-dominant & $0.84(0.56-1.27)$ & 0.41 \\
\hline \multirow[t]{2}{*}{ Intron4 } & $A / G$ & 50 & 41.3 & 53 & 31.7 & dominant & $0.71(0.44-1.15)$ & 0.17 \\
\hline & $\mathrm{G} / \mathrm{G}$ & 4 & 3.3 & 10 & 6.0 & recessive & $1.77(0.53-5.89)$ & 0.34 \\
\hline rs2024233 & $A / A$ & 38 & 31.4 & 49 & 29.3 & co-dominant & $1.11(0.79-1.57)$ & 0.53 \\
\hline \multirow[t]{2}{*}{ 3'-UTR } & $A / G$ & 63 & 52.1 & 85 & 50.9 & dominant & $1.10(0.66-1.83)$ & 0.72 \\
\hline & $\mathrm{G} / \mathrm{G}$ & 20 & 16.5 & 33 & 19.8 & recessive & $1.23(0.67-2.28)$ & 0.50 \\
\hline rs4730775 & $\mathrm{G} / \mathrm{G}$ & 70 & 57.9 & 106 & 63.9 & co-dominant & $0.87(0.59-1.28)$ & 0.48 \\
\hline \multirow[t]{2}{*}{ 3'-UTR } & $\mathrm{G} / \mathrm{A}$ & 45 & 37.2 & 49 & 29.5 & dominant & $0.76(0.47-1.24)$ & 0.27 \\
\hline & $\mathrm{A} / \mathrm{A}$ & 6 & 5.0 & 11 & 6.6 & recessive & $1.29(0.46-3.62)$ & 0.62 \\
\hline rs6948009 & $\mathrm{G} / \mathrm{G}$ & 87 & 73.1 & 124 & 74.7 & co-dominant & $0.80(0.48-1.33)$ & 0.39 \\
\hline \multirow[t]{2}{*}{ 3'-near gene } & $\mathrm{G} / \mathrm{A}$ & 29 & 24.4 & 42 & 25.3 & dominant & $0.89(0.52-1.53)$ & 0.67 \\
\hline & $\mathrm{A} / \mathrm{A}$ & 3 & 2.5 & 0 & 0 & recessive & $0.00(0.00-N A)$ & 0.02 \\
\hline
\end{tabular}

Freq, frequency; NPC, nonpoor concentration; PC, poor concentration; OR, odds ratio; Cl, confidence interval; N/A, not applicable.

www.ncbi.nlm.nih.gov/SNP dbSNP Build 130). This database presents genotype frequencies for the SNPs analyzed in this study (Table 6). The control group's genotype distributions of the SNPs we analyzed are similar to those of Asian populations, especially the Japanese population (Table 6).

\section{Discussion}

The WNT2 gene and its chromosomal location have received attention as a candidate gene with regard to autism. Given the similarities between autistic disorders and schizophrenia (as a neurodevelopmental disorder), we investigated whether WNT2 gene variations act as risk factors for schizophrenia in a Korean sample. The results suggest that WNT2 may not be involved in the pathogenesis of schizophrenia. Of all the SNPs and haplotypes analyzed, only one SNP (rs4730775) showed a weak association with the disorder. However, the significance disappeared after the Bonferroni correction for multiple testing $(\mathrm{p}=0.102$, Table 1$)$. In addition, we investigated the LD between the WNT2 SNPs and performed a haplotype analysis between the schizophrenia and control subjects. We could not find any association between WNT2 and schizophrenia, indicating that there is no genetic association between WNT2 and schizophrenia.

To further analyze the association test between clinical symptoms and SNP genotypes, we evaluated other symptoms, from the OPCRIT checklist and the total SANS 
Table 5: Comparison of SANS scores between schizophrenic patient groups with different genotypes of WNT2.

\begin{tabular}{|c|c|c|c|c|c|}
\hline \multirow[b]{2}{*}{ SNP } & \multirow[b]{2}{*}{ Genotype } & \multirow[b]{2}{*}{$\mathbf{n}$} & \multicolumn{3}{|c|}{ SANS } \\
\hline & & & Mean & S.E. & $P$ value \\
\hline rs39315 & $\mathrm{A} / \mathrm{A}$ & 90 & 66.78 & 2.627 & 0.388 \\
\hline \multirow[t]{2}{*}{ 5'-near gene } & $\mathrm{A} / \mathrm{G}$ & 137 & 63.76 & 2.239 & 0.866 \\
\hline & $\mathrm{G} / \mathrm{G}$ & 61 & 64.41 & 2.831 & 0.55 \\
\hline rs 17132543 & $\mathrm{~A} / \mathrm{A}$ & 97 & 63.99 & 2.46 & 0.458 \\
\hline \multirow[t]{2}{*}{ Intron4 } & $A / G$ & 138 & 66.51 & 2.256 & 0.278 \\
\hline & $\mathrm{G} / \mathrm{G}$ & 53 & 62.06 & 3.016 & 0.63 \\
\hline rs3779548 & $\mathrm{A} / \mathrm{A}$ & 127 & 66.42 & 2.139 & 0.689 \\
\hline \multirow[t]{2}{*}{ Intron4 } & $A / G$ & 128 & 65.17 & 2.257 & 0.124 \\
\hline & $\mathrm{G} / \mathrm{G}$ & 33 & 57.48 & 4.394 & 0.062 \\
\hline rs733154 & $\mathrm{A} / \mathrm{A}$ & 171 & 66.11 & 1.946 & 0.371 \\
\hline \multirow[t]{2}{*}{ Intron4 } & $A / G$ & 103 & 63.32 & 2.375 & 0.684 \\
\hline & $\mathrm{G} / \mathrm{G}$ & 14 & 60.5 & 6.791 & 0.429 \\
\hline rs2024233 & $\mathrm{A} / \mathrm{A}$ & 87 & 60.26 & 2.717 & 0.031 \\
\hline \multirow[t]{2}{*}{ 3'-UTR } & $A / G$ & 148 & 67.59 & 2.034 & 0.461 \\
\hline & $\mathrm{G} / \mathrm{G}$ & 53 & 64.68 & 3.319 & 0.311 \\
\hline rs4730775 & $\mathrm{G} / \mathrm{G}$ & 176 & 65.82 & 1.85 & 0.267 \\
\hline \multirow[t]{2}{*}{ 3'-UTR } & $\mathrm{G} / \mathrm{A}$ & 94 & 64.23 & 2.63 & 0.619 \\
\hline & $\mathrm{A} / \mathrm{A}$ & 17 & 58.82 & 6.56 & 0.427 \\
\hline rs6948009 & $\mathrm{A} / \mathrm{A}$ & 211 & 65.02 & 1.81 & 0.981 \\
\hline \multirow[t]{2}{*}{ 3'-near gene } & $A / G$ & 71 & 64.94 & 2.5 & 0.307 \\
\hline & $\mathrm{G} / \mathrm{G}$ & 3 & 52.33 & 3.38 & 0.406 \\
\hline
\end{tabular}

SANS, Scale for the Assessment of Negative Symptoms; S.E., Standard Error; SNP, single nucleotide polymorphism.

scores. However, we found no statistically significant association between any symptom in OPCRIT (Table 4) and total SANS score (Table 5). We compared our genotype frequencies with the human SNP database http:// www.ncbi.nlm.nih.gov/sites/entrez? $d b=s n p$, to show ethnic similarities and differences. The genotype frequencies of our study sample resemble those of the Japanese and Chinese Hapmap populations (Table 6). Further studies are necessary to elucidate (i) whether another case-control study is appropriate and (ii) whether promoter SNPs can affect the expression of WNT2. To confirm or refute the lack of association between the WNT2 gene and schizophrenia, replication studies with adequate sample sizes, or studies with additional SNPs not analyzed in the present study, may be required.
This report has several limitations. First, the sample size may not have been sufficient to detect associations of smaller effects on schizophrenia. We estimated that this sample had more than 0.8 powers to detect association, with the gene exerting a genotypic relative risk of approximately 2. However, schizophrenia appears to be a very genetically complex disease, and the WNT2 gene risks may be smaller than 2. Second, when we designed the experiment, the versions of the HapMap database and dbSNP database were slightly different compared to their present forms, and, as a result, our selection of SNPs provided incomplete coverage of currently-known common variations in the WNT2 gene. Furthermore, the present databases showed different locations for some SNP from 
Table 6: WNT2 SNPs genotype frequencies in each population

\begin{tabular}{|c|c|c|c|c|c|c|c|}
\hline SNP & Genotype & Schizophrenia & Control & Europe & China & Japan & $\begin{array}{c}\text { Sub-Saharan } \\
\text { African }\end{array}$ \\
\hline rs39315 & $\mathrm{A} / \mathrm{A}$ & 0.31 & 0.26 & 0.36 & 0.29 & 0.46 & 0.22 \\
\hline \multirow[t]{2}{*}{ 5'-near gene } & $A / G$ & 0.48 & 0.49 & 0.41 & 0.62 & 0.43 & 0.44 \\
\hline & $\mathrm{G} / \mathrm{G}$ & 0.21 & 0.25 & 0.22 & 0.09 & 0.11 & 0.34 \\
\hline$P$ value & & & & 0.285 & 0.010 & 0.003 & 0.373 \\
\hline rs17132543 & $\mathrm{A} / \mathrm{A}$ & 0.34 & 0.32 & 0.78 & 0.24 & 0.44 & 0.97 \\
\hline \multirow[t]{2}{*}{ lintron4 } & $A / G$ & 0.48 & 0.48 & 0.22 & 0.58 & 0.47 & 0.03 \\
\hline & $\mathrm{G} / \mathrm{G}$ & 0.18 & 0.20 & 0.00 & 0.18 & 0.09 & 0.00 \\
\hline$P$ value & & & & 0.000 & 0.334 & 0.048 & 0.000 \\
\hline rs3779548 & $\mathrm{A} / \mathrm{A}$ & 0.44 & 0.44 & 0.30 & 0.69 & 0.36 & 0.17 \\
\hline \multirow[t]{2}{*}{ Intron4 } & $\mathrm{A} / \mathrm{G}$ & 0.44 & 0.45 & 0.47 & 0.22 & 0.44 & 0.65 \\
\hline & $\mathrm{G} / \mathrm{G}$ & 0.11 & 0.11 & 0.23 & 0.09 & 0.20 & 0.18 \\
\hline$P$ value & & & & 0.031 & 0.001 & 0.180 & 0.000 \\
\hline rs733154 & $\mathrm{A} / \mathrm{A}$ & 0.59 & 0.58 & & 0.85 & 0.62 & 0.31 \\
\hline \multirow[t]{2}{*}{ Intron4 } & $A / G$ & 0.36 & 0.36 & No data & 0.13 & 0.31 & 0.37 \\
\hline & $\mathrm{G} / \mathrm{G}$ & 0.05 & 0.06 & & 0.03 & 0.08 & 0.33 \\
\hline$P$ value & & & & $\mathrm{N} / \mathrm{A}$ & 0.000 & 0.675 & 0.000 \\
\hline rs1051751 & $\mathrm{G} / \mathrm{G}$ & 1.00 & 1.00 & 1.00 & 1.00 & 0.98 & 1.00 \\
\hline Exon 5 & $\mathrm{G} / \mathrm{T}$ & 0.00 & 0.00 & 0.00 & 0.00 & 0.02 & 0.00 \\
\hline Cys294Phe & $T / T$ & 0.00 & 0.00 & 0.00 & 0.00 & 0.00 & 0.00 \\
\hline$P$ value & & & & N/A & $\mathrm{N} / \mathrm{A}$ & $\mathrm{N} / \mathrm{A}$ & N/A \\
\hline rs2024233 & $\mathrm{A} / \mathrm{A}$ & 0.30 & 0.28 & 0.50 & 0.29 & 0.34 & 0.54 \\
\hline \multirow[t]{2}{*}{ 3'-UTR } & $A / G$ & 0.51 & 0.51 & 0.40 & 0.44 & 0.50 & 0.41 \\
\hline & $\mathrm{G} / \mathrm{G}$ & 0.18 & 0.21 & 0.10 & 0.27 & 0.16 & 0.05 \\
\hline$P$ value & & & & 0.003 & 0.526 & 0.531 & 0.000 \\
\hline rs4730775 & $\mathrm{G} / \mathrm{G}$ & 0.61 & 0.60 & 0.37 & 0.71 & 0.62 & 0.46 \\
\hline \multirow[t]{2}{*}{ 3'-near gene } & $\mathrm{G} / \mathrm{A}$ & 0.33 & 0.37 & 0.36 & 0.27 & 0.29 & 0.45 \\
\hline & $\mathrm{A} / \mathrm{A}$ & 0.06 & 0.03 & 0.27 & 0.02 & 0.09 & 0.09 \\
\hline$P$ value & & & & 0.000 & 0.261 & 0.135 & 0.060 \\
\hline rs6948009 & $\mathrm{A} / \mathrm{A}$ & 0.74 & 0.53 & & 0.95 & 0.93 & \\
\hline \multirow[t]{2}{*}{ 3'-near gene } & $A / G$ & 0.25 & 0.45 & No data & 0.05 & 0.05 & No data \\
\hline & $\mathrm{G} / \mathrm{G}$ & 0.01 & 0.02 & & 0.00 & 0.02 & \\
\hline$P$ value & & & & $\mathrm{N} / \mathrm{A}$ & 0.000 & 0.000 & N/A \\
\hline
\end{tabular}

From database http://www.ncbi.nlm.nih.gov/sites/entrez?db=snp. We estimated all p-values via comparisons between the control group of our sample and each population. N/A, not applicable. 
previous databases. Consequently, we may have missed some of the relevant associations of WNT2 genes.

\section{Conclusions}

We investigated possible associations between WNT2 gene SNPs and schizophrenia, and the results were negative. For that reason, it appears the SNPs of WNT2 may not influence the development of schizophrenia in the Korean population, but, still, additional genetic studies will help develop understanding of the precise mechanisms underlying pathogenesis in schizophrenic patients.

\section{Competing interests}

The authors declare that they have no competing interests.

\section{Authors' contributions}

Authors JWK and JHC designed and directed the whole project. Authors SKK and SW K managed the literature searches and analyses. JYS, ARC, and JKP carried out the schizophrenia assessments and advised on patient selection. Author HKP advised on control group collection. Author HJK performed most of the statistical analyses and the genotyping and contributed substantially to the first draft of the manuscript. All authors contributed to and have approved the final manuscript

\section{Acknowledgements}

This study was supported by a grant of the Korea Healthcare technology R\&D project, Ministry for Health, Welfare \& Family Affairs, Republic of Korea (A090239)

\section{Author Details}

${ }^{1}$ College of Medicine, Soonchunhyang University, Chunan 336-745, Republic of Korea, ${ }^{2}$ Department of Neuropsychiatry, School of Medicine, Kyung Hee University, Seoul 130-701, Republic of Korea, ${ }^{3}$ Kohwang Medical Research Institute, School of Medicine, Kyung Hee University, Seoul 130-701, Republic of Korea and ${ }^{4}$ Department of Emergency Medicine, East-West Neo-media Center, Kyung Hee University, 149 Sang-il dong, Gangdong-gu, Seoul 134-727, Republic of Korea

Received: 7 October 2009 Accepted: 24 May 2010

Published: 24 May 2010

\section{References}

1. Freedman R: Schizophrenia. N Engl J Med 2003, 349:1738-1749.

2. Ross CA, Margolis RL, Reading SA, Pletnikov M, Coyle JT: Neurobiology of schizophrenia. Neuron 2006, 52:139-153.

3. Murray RM, Lewis SW: Is schizophrenia a neurodevelopmental disorder? BMJ 1987, 295:681-682.

4. Weinberger DR: Implications of normal brain development for the pathogenesis of schizophrenia. Arch Gen Psychiatry 1987, 44:660-669.

5. Bloom FE: Advancing a neurodevelopmental origin for schizophrenia. Arch Gen Psychiatry 1993, 50:224-227.

6. Lewis DA, Lieberman JA: Catching up on schizophrenia: Natural history and neurobiology. Neuron 2000, 28:325-334.

7. Brandon NJ, Millar JK, Korth C, Sive H, Singh KK, Sawa A: Understanding the role of DISC1 in psychiatric disease and during normal development. J Neurosci 2009, 29:12768-12775.

8. Freedman R, Goldowitz D: Studies on the hippocampal formation: From basic development to clinical applications: Studies on schizophrenia. Prog Neurobiol 2009 in press.

9. Potkin SG, Turner JA, Guffanti G, Lakatos A, Fallon JH, Nguyen DD, Mathalon D, Ford J, Lauriello J, Macciardi F, FBIRN: A genome-wide association study of schizophrenia using brain activation as a quantitative phenotype. Schizophr Bull 2009, 35:96-108.

10. Nusse R: Wnt signaling in disease and in development. Cell Res 2005, 15:28-32.

11. Kozlovsky N, Belmaker RH, Agam G: GSK-3 and the neurodevelopmental hypothesis of schizophrenia. Eur Neuropsychopharmacol 2002, 12:13-25.
12. Cotter D, Kerwin R, Al-Sarraji S, Brion JP, Chadwich A, Lovestone S: Abnormalities of Wnt signaling in schizophrenia - evidence for neurodevelopmental abnormality. Neuroreport 1998, 9:1379-1383.

13. Miyaoka T, Seno H, Ishino H: Increased expression of Wnt-1 in schizophrenic brains. Schizophr Res 1999, 38:1-6.

14. Emamian ES, Hall D, Birnbaum MJ, Karayiorgou M, Gogos JA: Convergent evidence for impaired AKT1-GSK3beta signaling in schizophrenia. Nat Genet 2004, 36:131-137.

15. Kozlovsky N, Belmaker RH, Agam G: Low GSK-3 activity in frontal cortex of schizophrenic patients. Schizophr Res 2001, 52:101-105.

16. Kozlovsky N, ShanonWeickert C, Tomaskovic-Crook E, Kleinman JE, Belmaker RH, Agam G: Reduced GSK-3betamRNAlevels in postmortem dorsolateral prefrontal cortex of schizophrenic patients. J Neural Transm 2004, 111:1583-1592.

17. Katsu T, Ujike H, Nakano T, Tanaka Y, Nomura A, Nakata K, Takaki M, Sakai A, Uchida N, Imamura T, Kuroda S: The human frizzled-3 (FZD3) gene on chromosome $8 \mathrm{p} 21$, a receptor gene for Wnt ligands, is associated with the susceptibility to schizophrenia. Neurosci Lett 2003, 353:53-56.

18. Glatt SJ, Wang RS, Yeh YC, Tsuang MT, Faraone SV: Five NOTCH4 polymorphisms show weak evidence for association with schizophrenia: evidence from meta-analyses. Schizophr Res 2005, 73:281-290

19. Jeong SH, Joo EJ, Ahn YM, Lee KY, Kim YS: Investigation of genetic association between human Frizzled homolog 3 gene (FZD3) and schizophrenia: Results in a Korean population and evidence from metaanalysis. Psychiatry Res 2006, 143:1-11.

20. Cotter D, Kerwin R, Al-Sarraji S, Brion JP, Chadwich A, Lovestone S, Anderton B, Everall I: Abnormalities of Wnt signalling in schizophrenia evidence for neurodevelopmental abnormality. Neuroreport 1998 9:1261-1265.

21. Lovestone S, Killick R, Forti MD, Murray R: Schizophrenia as a GSK-3 dysregulation disorder. Trends Neurosci 2007, 30:142-149.

22. Emamian ES, Hall D, Birnbaum MJ, Karayiorgou M, Gogos JA: Convergent evidence for impaired AKT1-GSK3b signaling in schizophrenia. Nat Genet 2004, 36:131-137.

23. Miyaoka T, Seno H, Ishino H: Increased expression of Wnt-1 in schizophrenic brains. Schizophr Res 1999, 38:1-6.

24. Yang J, Si T, Ling Y, Ruan Y, Han Y, Wang X, Zhang H, Kong Q, Li X, Liu C, Zhang D, Zhou M, Yu Y, Liu S, Shu L, Ma D, Wei J, Zhang D: Association study of the human FZD3 locus with schizophrenia. Biol Psychiatry 2003, 54:1298-1301.

25. Zhang Y, Yu X, Yuan Y, Ling Y, Ruan Y, Si T, Lu T, Wu S, Gong X, Zhu Z, et al:: Positive association of the human frizzled 3 (FZD3) gene haplotype with schizophrenia in Chinese Han population. Am J Med Genet B Neuropsychiatr Genet part B 2004, 129:16-19.

26. Katsu T, Ujike H, Nakano T, Tanaka Y, Nomura A, Nakata K, Takaki M, Sakai A, Uchida N, Imamura T, Kuroda S: The human frizzled-3 (FZD3) gene on chromosome $8 \mathrm{p} 21$, a receptor gene for Wnt ligands, is associated with the susceptibility to schizophrenia. Neurosci Lett 2003, 353:53-56.

27. Faraone SV, Matise T, Svrakic D, Pepple J, Malaspina D, Suarez B, Hampe C, Zambuto CT, Schmitt K, Meyer J, Markel P, Lee H, Harkavy Friedman J, Kaufmann C, Cloninger CR, Tsuang MT: Genome scan of EuropeanAmerican schizophrenia pedigrees: results of the NIMH Genetics Initiative and Millennium Consortium. Am J Med Genet 1998, 81:290-295.

28. Cadigan KM, Nusse R: Wnt signaling: a common theme in animal development. Genes Dev 1997, 11:3286-3305.

29. Uusitalo M, Heikkila M, Vainio S: Molecular genetic studies of Wnt signaling in the mouse. Exp Cell Res 1999, 253:336-348

30. Hauptmann G, Gerster T: Regulatory gene expression patterns revea transverse and longitudinal subdivisions of the embryonic zebra fish forebrain. Mech Dev 2000, 91:105-118

31. Landesman Y, Sokol SY: Xwnt-2b is a novel axis-inducing Xenopus Wnt, which is expressed in embryonic brain. Mech Dev 1997, 63:199-209.

32. Brown JD, Moon RT: Wnt signaling: why is everything so negative? Curr Opin Cell Biol 1998, 10:182-187.

33. Wodarz A, Nusse R: Mechanisms of Wnt signaling in development. Ann Rev Cell Dev Biol 1998, 14:59-88.

34. Salinas PC: Wnt factors in axonal remodelling and synaptogenesis. Biochem Soc Symp 1999, 65:101-109. 
35. Wassink TH, Piven J, Vieland VJ, Huang J, Swiderski RE, Pietila J, Braun T, Beck G, Folstein SE, Haines JL, Sheffield VC: Evidence supporting WNT2 as an autism susceptibility gene. Am J Med Genet 2001, 105:406-413.

36. Li J, Nguyen L, Gleason C, Lotspeich L, Spiker D, Risch N, Myers RM: Lack of evidence for an association between WNT2 and RELN polymorphisms and autism. Am J Med Genet B Neuropsychiatr Genet 2004, 126B:51-57.

37. McCoy PA, Shao Y, Wolpert CM, Donnelly SL, Ashley-Koch A, Abel HL, Ravan SA, Abramson RK, Wright HH, DeLong GR, Cuccaro ML, Gilbert JR, Pericak-Vance MA: No association between the WNT2 gene and autistic disorder. Am J Med Genet 2002, 114:106-109.

38. Proitsi P, Li T, Hamilton G, Di Forti M, Collier D, Killick R, Chen R, Sham P, Murray R, Powell J, Lovestone S: Positional pathway screen of wnt signaling genes in schizophrenia: association with DKK4. Biol Psychiatry 2008, 63:13-16

39. American Psychiatric Association: Diagnostic and statistical manual of mental disorders 4th edition. Washington DC: American Psychiatric Press; 1994.

40. Flemenbaum A, Zimmermann RL: Inter- and intra-rater reliability of the brief psychiatric rating scale. Psychological Reports 1973, 32:783-792.

41. Andreasen NC: Negative symptoms in schizophrenia. Definition and reliability. Archives of General Psychiatry 1982, 39:784-788.

42. McGuffin P, Farmer A, Harvey I: A polydiagnostic application of operational criteria in studies of psychotic illness. Development and reliability of the OPCRIT system. Arch Gen Psychiatry 1991, 48:764-770.

43. Kim HJ, Park HJ, Jung KH, Ban JY, Ra J, Kim JW, Park JK, Choe BK, Yim SV, Kwon YK, Chung JH: Association study of polymorphisms between DISC1 and schizophrenia in a Korean population. Neurosci Lett 2008, 430:60-63.

44. Rickham PP: Human experimentation. Code of ethics of the world medical association. Declaration of helsinki. British Medical Journal 1964, 2:177.

45. Solé X, Guinó E, Valls J, Iniesta R, Moreno V: SNPStats: a web tool for the analysis of association studies. Bioinformatics 2006, 22:1928-1929.

46. Barrett JC, Fry B, Maller J, Daly MJ: Haploview: analysis and visualization of LD and haplotype maps. Bioinformatics 2005, 21:263-265.

47. Stephens M, Smith NJ, Donnelly P: A new statistical method for haplotype reconstruction from population data. Am J Hum Genet 2001, 68:78-89.

48. Jung HY, Park JS, Park YJ, Kim YK, Kimm K, Koh IS: HapAnalyzer: Minimum Haplotype Analysis System for Association Studies. Genomics \& Informatics 2004, 2:107-109.

49. Purcell S, Cherny SS, Sham PC: Genetic Power Calculator: design of linkage and association genetic mapping studies of complex traits. Bioinformatics 2003, 19:149-150.

50. Gabriel SB, Schaffner SF, Nguyen H, Moore JM, Roy J, Blumenstiel B, Higgins J, DeFelice M, Lochner A, Faggart M, Liu-Cordero SN, Rotimi C, Adeyemo A, Cooper R, Ward R, Lander ES, Daly MJ, Altshuler D: The structure of haplotype blocks in the human genome. Science 2002, 296:2225-2229.

Pre-publication history

The pre-publication history for this paper can be accessed here: http://www.biomedcentral.com/1471-2350/11/78/prepub

doi: $10.1186 / 1471-2350-11-78$

Cite this article as: Kim et al., No association between polymorphisms of WNT2 and schizophrenia in a Korean population BMC Medical Genetics 2010, $11: 78$

Submit your next manuscript to BioMed Central and take full advantage of:

- Convenient online submission

- Thorough peer review

- No space constraints or color figure charges

- Immediate publication on acceptance

- Inclusion in PubMed, CAS, Scopus and Google Scholar

- Research which is freely available for redistribution 